\title{
Fourth-generation Synchrotron radiation and XFEL facilities: new era in crystallography and material science
}

\author{
Alexander Eugenievich Blagov \\ National Research Center «Kurchatov Institute», Moscow, Russian Federation; \\ blagov_ae@mail.ru
}

The capabilities of 4th generation synchrotron radiation sources and X-ray free electron lasers such as high brightness, coherence and temporal structure of pulses open new horizons in the studies of structure, structural dynamics and properties of materials.

$\mathrm{X}$-ray radiation coherent methods enable to get access to the 3D structure of non-crystalline samples, nanocrystals and nanostructures with a resolution theoretically limited only by the diffraction limit [1]. Such samples include, for example, various biological objects [2], biological cells, viruses and nanosized crystallites of bio macromolecules and their complexes which are difficult to crystallize.

Access to an atomic structure with ultra-high temporal resolution using ultrashort pulses of free electron lasers makes it possible to consider the different tasks of studying chemical reactions, self-organization and destruction of materials mechanisms, the formation of short-range and long-range orders, the study of phase transitions, and the complex dynamics of proteins and polymers at a fundamentally new level.

Novel scientific tasks cover a wide range of practical applications [3], including such priority areas as biotechnology and medicine, the creation of new functional materials (structural, composite, etc.), nanoelectronics and hybrid (sensors, biosensors, etc.).

Today most of the new synchrotron radiation sources have almost $100 \%$ transverse coherence, and the modernization of existing mega-facilities (for example, ESRF-EBS, PETRA IV, APS) focus on reducing the emittance (significantly less than $1 \mathrm{~nm}$ ), increasing coherence, brightness and time resolution.

In the Russian Federation the development of coherent scattering and time resolving methods is becoming one of the priority tasks in connection with the implementation of the program for the development of the synchrotron-neutron infrastructure, including the construction of 4th generation sources: USSR-4 (synchrotron with a free electron laser) and SKIF project.

[1] J. Miao, T. Ishikawa, I. Robinson, M. Murnane Beyond crystallography: Diffractive imaging using coherent x-ray light sources. Science. 2015. V. 348. 6234. P. 530.

[2] A. Mancuso, O. Yefanov, I. Vartanyants, Coherent diffractive imaging of biological samples at synchrotron and free electron laser facilities Journal of Biotechnology, Volume 149, Issue 4, 2010, P. 229.

[3] H.Chapman, K. Nugent Coherent lensless X-ray imaging. Nature Photonics. 2010. V. 4. P. 833.

Keywords: 4th generation SR and XFEL facilities, coherent diffraction and scattering 